\title{
Contribuições no processo de ensino aprendizagem da prática de simulação
}

\section{realística: uma revisão sistemática}

\author{
Contributions to the teaching process learning the practice of realistic simulariam: an sistematic \\ review \\ Contribuciones al proceso de enseñanza aprendiendo la práctica de la simulácion realista: una \\ revisión sistemática
}

\author{
Mariana Da Costa Daniel \\ ORCID: https://orcid.org/0000-0001-5795-7338 \\ Universidade do Estado do Rio de Janeiro, Brasil \\ E-mail: costadmari@gmail.com \\ Joyce Fernandes Rodrigues Galante \\ ORCID: https://orcid.org/0000-0002-0007-9594 \\ Universidade do Estado do Rio de Janeiro, Brasil \\ E-mail:joynandes1996@gmail.com \\ Juliana Assumpção de Paiva Furtado \\ ORCID: https://orcid.org/0000-0002-7937-8617 \\ Universidade do Estado do Rio de Janeiro, Brasil \\ E-mail: enf.julianafurt@gmail.com \\ Myllena Leal da Silva \\ ORCID: https://orcid.org/0000-0003-1903-8909 \\ Universidade Castelo Branco, Brasil \\ E-mail: myllenaleal42@gmail.com \\ Paloma Vitória Serra Batista \\ ORCID: https://orcid.org/0000-0002-7591-0792 \\ Universidade do Estado do Rio de Janeiro, Brasil \\ E-mail: paloma.vitoriaserra@hotmail.com \\ Raquel Freitas de Souza \\ ORCID: https://orcid.org/0000-0003-1562-6439 \\ Universidade do Estado do Rio de Janeiro, Brasil \\ E-mail: raquelfreitasdsouza@gmail.com \\ Alessandra Sant Anna Nunes \\ ORCID: https://orcid.org/0000-0001-7435-2568 \\ Universidade Federal do Estado do Rio de Janeiro, Brasil \\ E-mail: asantnunes@gmail.com \\ Advi Catarina Barbachan Moraes \\ ORCID: https://orcid.org/0000-0002-1470-2623 \\ Universidade do Estado do Rio de Janeiro, Brasil \\ E-mail: Advicbmoraes@gmail.com
}

\section{Resumo}

Objetivos: Analisar as contribuições da simulação realística em contraposição ao ensino tradicional da graduação de enfermagem. Objetivos específicos: visam descrever as vantagens da simulação realística como estratégia de ensino para a formação de enfermeiros; identificar as técnicas da simulação realística utilizadas como estratégia de ensino para o curso de enfermagem e identificar a eficácia do uso da simulação realística no processo de ensinoaprendizagem. Método: Trata-se de um estudo descritivo do tipo revisão sistemática de literatura. Foram analisados uma amostra final de 17 estudos publicados nos últimos cinco anos nas seguintes bases de dados MEDLINE, LILACS e BDENF. Tendo como população os alunos da graduação de enfermagem. Resultados: A simulação realística, ao contrário do ensino tradicional, permite que se alcance uma aproximação entre a realidade e a teoria, auxiliando na construção de habilidades, conhecimentos e diminuindo os riscos. Contribui para que o aprendiz desenvolva autonomia, confiança e senso crítico em suas práticas clínicas. É uma melhor estratégia de aprendizagem, visto que agrega diversos benefícios ao estudante através da aplicação da metodologia ativa.

Palavras-chave: Estudantes de enfermagem; Simulação de paciente; Treinamento em simulação. 


\begin{abstract}
Objectives: To analyze the contributions of realistic simulation as opposed to traditional nursing undergraduate education. Specific objectives: aim to describe the advantages of realistic simulation as a teaching strategy for nursing education; identify the realistic simulation techniques used as a teaching strategy for the nursing course and identify the effectiveness of using realistic simulation in the teaching-learning process. Method: This is a descriptive study of the systematic literature review type. A final sample of 17 studies published in the last five years in the following databases MEDLINE, LILACS and BDENF was analyzed. With the population of undergraduate nursing students. Results: Realistic simulation, unlike traditional teaching, allows for an approximation between reality and theory, helping to build skills, knowledge and reducing risks. It helps the apprentice to develop autonomy, confidence and critical thinking in their clinical practices. It is a better learning strategy, as it adds several benefits to the student through the application of the active methodology.
\end{abstract}

Keywords: Nursing students; Patient simulation; Simulation training.

\title{
Resumen
}

Objetivos: Analizar los aportes de la simulación realista frente a la formación tradicional de pregrado en enfermería. Objetivos específicos: tratar de describir las ventajas de la simulación realista como estrategia de enseñanza para la formación en enfermería; identificar las técnicas de simulación realista utilizadas como estrategia de enseñanza para el curso de enfermería e identificar la efectividad de utilizar la simulación realista en el proceso de enseñanzaaprendizaje. Método: Se trata de un estudio descriptivo del tipo revisión sistemática de la literatura. Se analizó una muestra final de 17 estudios publicados en los últimos cinco años en las siguientes bases de datos MEDLINE, LILACS y BDENF. Teniendo como población a los estudiantes de licenciatura en enfermería. Resultados: La simulación realista, a diferencia de la enseñanza tradicional, permite una aproximación entre la realidad y la teoría, ayudando a construir habilidades, conocimientos y reduciendo riesgos. Ayuda al aprendiz a desarrollar autonomía, confianza y pensamiento crítico en sus prácticas clínicas. Es una mejor estrategia de aprendizaje, ya que agrega varios beneficios al alumno mediante la aplicación de la metodología activa.

Palabras clave: Estudiantes de enfermería; Simulación de pacientes; Entrenamiento de simulación.

\section{Introdução}

A simulação e o treinamento por simulação são considerados estratégias de ensino cruciais na formação de profissionais de saúde, e têm como característica causar impactos em diversos aspectos, desde a satisfação à segurança pessoal até os objetivos de cuidado em saúde. (Martins, 2017). Além disso, é um processo de educação cognitiva e de comportamento, pois podem proporcionar um aumento na autoestima e autoconfiança, o que amplia a possibilidade dos indivíduos absorverem informações e obterem benefícios no processo de aprendizagem. (Miranda et al., 2018).

Por um lado, historicamente busca-se evoluir as técnicas de cuidado de acordo com o aparecimento de novas tecnologias, e inserido no contexto atual, a implementação de simulação realística nos cursos de graduação tem sido cada vez mais buscada entre as instituições. Essa nova tecnologia de ensino permite que se alcance uma aproximação entre a realidade e a teoria, construindo conhecimento e habilidades técnicas reduzindo os riscos. Além disso, esse cenário de ensinoaprendizagem permite o desenvolvimento de trabalho em equipe e espíritos de liderança os quais serão fundamentais no processo de cuidado com o paciente. Sendo assim, novas tecnologias de aprendizado estão sempre em construção visando aprimorar a qualidade de ensino. (Dourado et al., 2014).

Embora a prática pedagógica tenha sido repensada por muitos autores constantemente, Paulo Freire como um educador e filósofo também se aprofundou nesse tema e descreveu o que chamou de educação bancária, a qual consiste no processo de verticalização da educação, onde há a hierarquia de autoridade entre educador e educando. Nesse caso, o educador é o detentor do saber, e subordinado a ele está o educando, o qual irá absorver toda a informação passada por seu educador, geralmente de forma oral, sem que haja troca de críticas e reflexões entre ambos, assim excluindo a autonomia do educando no processo de ensino-aprendizagem. Essa prática pedagógica é uma herança dos primórdios jesuítas, os quais foram os primeiros educadores, que criou um caráter tradicional no método educativo, sendo o mais adotado pelas sociedades. (Brighente et al., 2016).

Também considerando que o método tradicional de educação utilizado seja a educação bancária, descrita por Paulo 
Freire, o que se encontra muito nas práticas educativas na graduação em enfermagem é a continuidade desse sistema de depósito de informações do docente aos alunos, sendo assim, majoritariamente, não são implementadas novas metodologias de ensino, seja por falta de conhecimento docente diante de novas tecnologias educativas ou por característica própria do cenário educacional da instituição. (Gadelha et al., 2020).

Contrariamente na metodologia ativa, o aluno é o próprio sujeito ativo do processo de ensino-aprendizagem. Essa metodologia está sempre em transformação e engloba o ensino, a pesquisa e a assistência. Além disso, estimula o estudante a solucionar problemas e ter um pensamento crítico, desenvolvendo sua autonomia. A metodologia ativa faz uma grande contraposição com a forma tradicional de aprendizagem, ela estimula o aluno a pensar, já na forma tradicional há um distanciamento do educador, tornando-a desestimulante. (Veiga et al., 2020).

Adicionalmente, dentro do princípio da educação ativa, existe a simulação realística, que é um método de ensino inovador, que serve para promover ao aluno um ensino de habilidades eficaz, desenvolvendo nele também confiança e autonomia em sua prática clínica. A simulação além de promover habilidade motora também lhe dá competência para a tomada de decisão, e faz com que os alunos possam desenvolver um senso crítico nas situações propostas, fazendo com que ele aprenda a lidar com a pressão e a resolução de problemas. (Silva et al., 2020)

Por outro lado, o primeiro experimento que se assemelha a simulação clínica ocorreu em Paris no século XVIII, onde um pai e filho que se chamam Grégories, elaboraram uma pelve feminina para ser usada no ensino de procedimentos obstétricos. Essa atuação levou a diminuição da mortalidade materna-infantil. (Rosen, 2008). Depois o primeiro exemplo de simulação manifestou-se na área aeroespacial, através da criação em 1929 da "Blue Box", que visa reproduzir os movimentos e sensações ocorridas em um voo. (Edwin, 2014).

Da mesma maneira, em 1960 Ausmund Laerdal desenvolveu um modelo de simulação com a intenção de ensinar como deve ser feita a ventilação boca a boca, no qual foi chamado Ressuci-Anne, favorecendo assim o treinamento das técnicas de ventilação em situações de emergência. (Cooper et al., 2008; Rosen, 2008). Posteriormente, em 1968, no American Heart Association Scientific Sessions, pelo Dr. Michael Gordon, da Universidade de Miami Medical School o primeiro manequim que se tem registro de tamanho completo chamado Harvey, sendo um simulador de paciente cardiológico, onde 27 doenças cardíacas eram simuladas. Esse foi o primeiro exemplo moderno de um simulador. Logo, conseguia achar diversos achados físicos, incluindo pressão arterial, ausculta, veias, artérias com pulsos, impulsos precordiais e variavam de acordo com a respiração. (Gaba, 2004).

Entretanto, muitos dos enfermeiros que se formaram no Brasil nas décadas de 70 e 80 , executaram nessa época as coletas sanguíneas como sendo seus primeiros procedimentos invasivos em um enfermo, a partir disso passou-se a realizar procedimentos mais complexos em humanos. Tendo em vista que o ambiente de ensino de enfermagem não possuía equipamentos de simulação e instalações para os procedimentos na realização das práticas de cuidado. (Martins, 2012).

Deste modo, a combinação da busca pela excelência científica e eficiência técnica, tem promovido a reorganização dos cursos de enfermagem, voltados para uma formação de qualidade. Tendo como objetivo formar enfermeiros generalistas, críticos, empáticos, competentes na prática, atendendo às necessidades de saúde social, com foco no Sistema Único de Saúde (SUS) e os princípios da ética e da segurança. (Konh et al., 1999).

Portanto, a simulação realística possui como principal objetivo reduzir erros preveníveis, montando cenários com situações do cotidiano em saúde em um ambiente seguro no qual admite-se erros sem provocar danos a nenhum paciente. Através dessa metodologia, é possível um melhor entendimento sobre os conceitos teóricos adquiridos pela forma tradicional de educação e, proporciona também, um aperfeiçoamento de habilidades, uma vez que é permitido uma repetição do treinamento. Essa possibilidade contribui para uma melhora na autoconfiança e segurança por parte dos alunos, uma vez que já desenvolveram um conhecimento e um treinamento prévio antes de ter que realizar a prática efetivamente com algum paciente. 
(Barreto et al., 2014; Yamane et al., 2019).

Além disso, a simulação realística é muito versátil, ou seja, é possível tanto a criação de cenários considerados mais simples como uma consulta clínica quanto a criação de cenários mais complexos, os quais requerem métodos mais invasivos como por exemplo uma cirurgia. (Yamane et al., 2019).

Considerando o tema proposto, neste estudo a questão norteadora é a seguinte interrogativa: Quais as contribuições no processo de ensino aprendizagem causadas pela prática de simulação realística em comparação ao ensino tradicional em alunos da graduação de enfermagem? A justificativa está sustentada na possibilidade de um levantamento de um referencial teórico atualizado sobre o emprego da metodologia de simulação realística como um fator contribuinte para formação de enfermeiros em comparação ao ensino tradicional. A fim de subsidiar uma educação baseada em evidências e com segurança, oferecendo aos discentes um maior empenho e alcance de conhecimentos mais sedimentados. Delimita-se, como objetivo geral: analisar as contribuições da simulação realística em contraposição ao ensino tradicional da graduação de enfermagem. E os objetivos específicos visam descrever as vantagens da simulação realística como estratégia de ensino para a formação de enfermeiros; identificar as técnicas da simulação realística utilizadas como estratégia de ensino para o curso de enfermagem e identificar a eficácia do uso da simulação realística no processo de ensino-aprendizagem.

\section{Metodologia}

O presente estudo trata-se de uma revisão sistemática de literatura, buscando colher uma síntese de informações a respeito da temática, reunindo diversas fontes de dados de uma maneira sistematizada. (Mendes, et al., 2008). Sendo assim, realizou-se uma pergunta PICO, o qual seria um acrônimo utilizado para População(P), Intervenção(I), Comparação(C) e Desfecho(O). A pergunta PICO utilizada para análise dos artigos foi "Quais as contribuições no processo de ensino aprendizagem causadas pela prática de simulação realística em comparação ao ensino tradicional em alunos da graduação de enfermagem?" Em que o "P" foram os alunos de graduação de enfermagem; o "I" a prática de simulação realística; o "C" referente ao ensino tradicional e o "O” são as contribuições no processo de ensino aprendizagem causadas pela prática de simulação realística (Akobeng AK, 2005).

A seleção inicial dos artigos foi realizada a partir da leitura dos títulos e posteriormente pela análise de seus resumos nas bases de dados MEDLINE, LILACS e BDENF. Essa pesquisa foi realizada no período de janeiro a fevereiro de 2021, cujo critérios de inclusão utilizados foram textos disponíveis online, estudos publicados nos últimos 5 anos, nos idiomas inglês, português e espanhol e, os tipos de estudos foram: ensaio clínico controlado, estudo de avaliação, guia de prática clínica e estudo observacional. Foram utilizados os seguintes termos baseados nos Descritores em Ciências da Saúde (DeCs): “Treinamento por simulação" e "Educação em enfermagem". A primeira busca recuperou 725 trabalhos, ao aplicar os filtros de inclusão por ano, idioma e tipo de estudo, foram encontrados respectivamente 536, 527 e por fim 94 artigos.

Com isso, os critérios de exclusão foram: publicações duplicadas, trabalhos que não apresentaram o uso da estratégia da simulação e pesquisas não realizadas com estudantes de graduação em Enfermagem. Após a leitura de títulos e resumos, do total de 94 artigos selecionados na Biblioteca Virtual em Saúde (BVS) foram retirados 78, obtendo-se uma amostra final composta de 16 estudos, sendo 12 da MEDLINE e 4 da LILACS e BDENF-enfermagem. Foi adicionado um novo artigo por meio da Biblioteca Eletrônica Científica Online (SCIELO), o qual se encaixou dentro dos critérios de inclusão do atual trabalho, e assim, contabilizou-se 17 estudos na seleção. Na Figura 1 descreve-se, em forma de fluxograma, os critérios de inclusão e exclusão aplicados. 


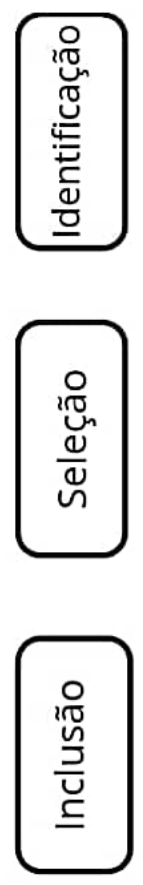

Figura 1. Fluxograma dos critérios de inclusão e exclusão.

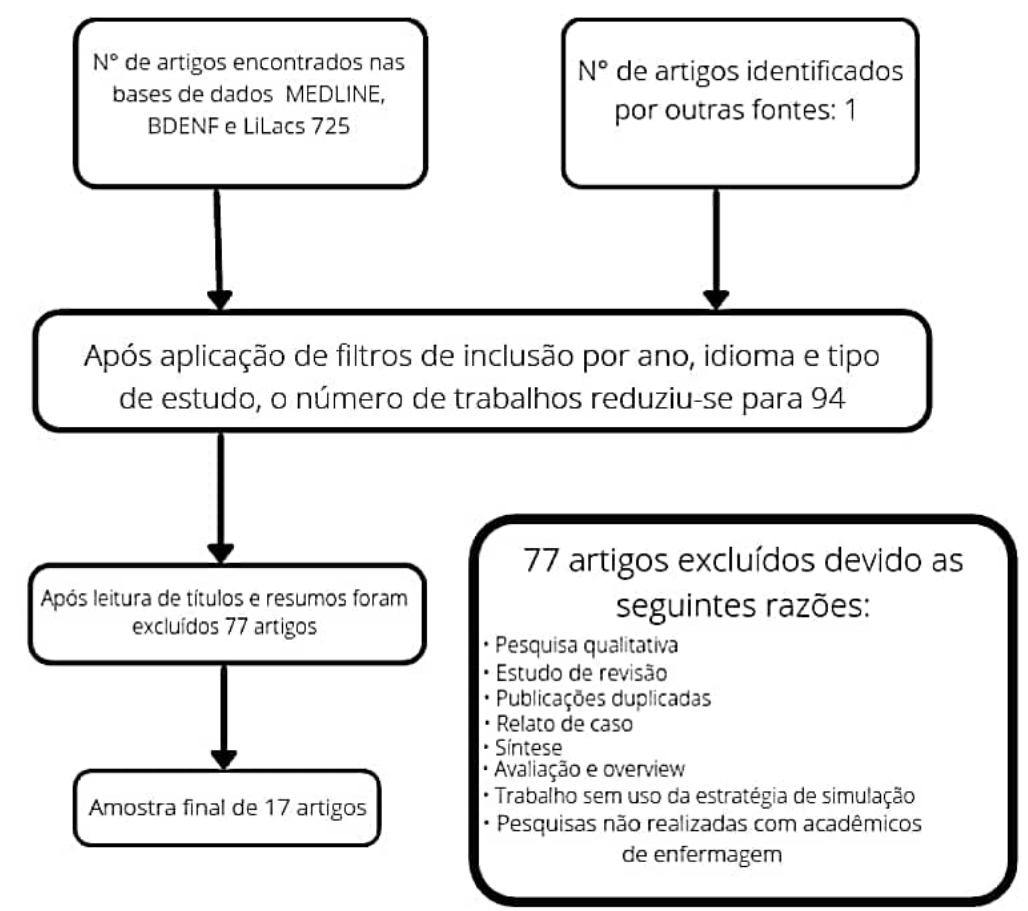

Fonte: Autores (2021).

Os artigos foram lidos na íntegra e organizados em uma planilha eletrônica com o autor, título do artigo, revista, ano de publicação e língua. Os estudos foram identificados com código numérico de 1 a 17 . Além disso, foi realizado um questionário com perguntas que serviram como base para a coleta de dados nos artigos. O Quadro 1 elucida os parâmetros acima descritos.

Quadro 1: Estudos sobre o uso da simulação na formação de enfermeiros segundo autor, título, periódico e língua nos anos de 2016-2020.

\begin{tabular}{|c|c|c|c|c|c|}
\hline $\mathbf{N}^{\circ}$ & Autor & Título & Períodico & Ano & Língua \\
\hline 1 & $\begin{array}{l}\text { Costa, R. R. O., Medeiros, S. M., } \\
\text { Martins, J. C. A., Coutinho, V. R. } \\
\text { D., \& Araújo, M. S. }\end{array}$ & $\begin{array}{l}\text { Eficácia da simulação no ensino de } \\
\text { imunização em enfermagem: ensaio clínico } \\
\text { randomizado }\end{array}$ & $\begin{array}{l}\text { RLA (Revista } \\
\text { Latino- } \\
\text { Americana de } \\
\text { Enfermagem) }\end{array}$ & 2020 & Português \\
\hline 2 & $\begin{array}{l}\text { Costa, R. R. O., Medeiros, S. M., } \\
\text { Coutinho V. R. D., Mazzo, A., \& } \\
\text { Araújo, M. S. }\end{array}$ & $\begin{array}{l}\text { Satisfação e autoconfiança na aprendizagem } \\
\text { de estudantes de enfermagem: Ensaio clínico } \\
\text { randomizado }\end{array}$ & $\begin{array}{l}\text { EAN (Escola } \\
\text { Anna Nery) }\end{array}$ & 2020 & $\begin{array}{l}\text { Português } \\
\text { e inglês }\end{array}$ \\
\hline 3 & $\begin{array}{l}\text { Curl, E. D., Smith, S., Chisholm, } \\
\text { L. A., McGee, L. A., \& Das, K. }\end{array}$ & $\begin{array}{l}\text { Effectiveness of Integrated Simulation and } \\
\text { Clinical Experiences Compared to Traditional } \\
\text { Clinical Experiences for Nursing Students }\end{array}$ & $\begin{array}{l}\text { Nursing } \\
\text { Education } \\
\text { Perspectives }\end{array}$ & 2016 & Inglês \\
\hline 4 & $\begin{array}{l}\text { Kuo, S., Wu , J., Chen, H., Chen, } \\
\text { C., \& Hu, S. H. }\end{array}$ & $\begin{array}{l}\text { Comparison of the effects of simulation } \\
\text { training and problem-based scenarios on the } \\
\text { improvement of graduating nursing students } \\
\text { to speak up about medication errors: A quasi- } \\
\text { experimental study }\end{array}$ & $\begin{array}{l}\text { Nurse } \\
\text { Education } \\
\text { Today }\end{array}$ & 2020 & Inglês \\
\hline 5 & $\begin{array}{l}\text { Bortolato-Majo, C., Mantovani, } \\
\text { M. d. F., Felix, J. V. C., Boostel, } \\
\text { R., \& Mattei, S. H }\end{array}$ & $\begin{array}{l}\text { Autoconfiança e satisfação dos estudantes de } \\
\text { enfermagem em simulação de emergência }\end{array}$ & $\begin{array}{l}\text { Reme - Revista } \\
\text { Mineira De } \\
\text { Enfermagem }\end{array}$ & 2020 & Português \\
\hline 6 & $\begin{array}{l}\text { Boostel, R., Felix, J., Bortolato- } \\
\text { Major, C., Pedrolo, E., \& Vayego, } \\
\text { S. }\end{array}$ & $\begin{array}{l}\text { Estresse do estudante de enfermagem na } \\
\text { simulação clínica: ensaio clínico } \\
\text { randomizado }\end{array}$ & $\begin{array}{l}\text { Reben - Revista } \\
\text { Brasileira De } \\
\text { Enfermagem }\end{array}$ & 2017 & Português \\
\hline 7 & $\begin{array}{l}\text { Salgado, P. O., Souza, C. C., } \\
\text { Júnior, P. P. P., Balbino, P. C., } \\
\text { Ribeiro, L., Paiva, L. C., \& } \\
\text { Brombine, N. L. M. }\end{array}$ & $\begin{array}{l}\text { O uso da simulação no ensino da técnica de } \\
\text { aspiração de vias aéreas: ensaio clínico } \\
\text { randomizado controlado }\end{array}$ & $\begin{array}{l}\text { REME - Rev } \\
\text { Min Enferm. }\end{array}$ & 2018 & $\begin{array}{l}\text { Português } \\
\text { e inglês }\end{array}$ \\
\hline
\end{tabular}




\begin{tabular}{|c|c|c|c|c|c|}
\hline 8 & $\begin{array}{l}\text { Alamrani, M. H., Alammar, K. } \\
\text { A., Alqahtani, S. S., \& Salem, O. } \\
\text { A. }\end{array}$ & $\begin{array}{l}\text { Comparing the Effects of Simulation-Based } \\
\text { and Traditional Teaching Methods on the } \\
\text { Critical Thinking Abilities and Self- } \\
\text { Confidence of Nursing Students }\end{array}$ & $\begin{array}{l}\text { Journal of } \\
\text { Nursing } \\
\text { Research }\end{array}$ & 2018 & Inglês \\
\hline 9 & Gray, B., \& Canver, J. & $\begin{array}{l}\text { Using Simulation to Enhance Education in an } \\
\text { Obstetrical } \\
\text { Nursing Course }\end{array}$ & $\begin{array}{l}\text { Nursing } \\
\text { Education } \\
\text { Perspectives }\end{array}$ & 2017 & Inglês \\
\hline 10 & $\begin{array}{l}\text { Baptista, R. C. N., Paiva, L. A. } \\
\text { R., Gonçalves, R. F. L., Oliveira, } \\
\text { L. M. N., Pereira, M. F. C. R., \& } \\
\text { Martins, J. C. A. }\end{array}$ & $\begin{array}{l}\text { Satisfaction and gains perceived by nursing } \\
\text { students with medium and high-fidelity } \\
\text { simulation: A randomized controlled trial }\end{array}$ & $\begin{array}{l}\text { Nurse } \\
\text { Education } \\
\text { Today }\end{array}$ & 2016 & Inglês \\
\hline 11 & Son, H. K. & $\begin{array}{l}\text { Effects of S-PBL in Maternity Nursing } \\
\text { Clinical Practicum on Learning Attitude, } \\
\text { Metacognition, and Critical Thinking in } \\
\text { Nursing Students: A Quasi-Experimental } \\
\text { Design. }\end{array}$ & $\begin{array}{l}\text { International } \\
\text { Journal of } \\
\text { Environmental } \\
\text { Research and } \\
\text { Public Health }\end{array}$ & 2020 & Inglês \\
\hline 12 & Page-Cutrara, K., \& Turk, M. & $\begin{array}{l}\text { Impact of prebriefing on competency } \\
\text { performance, clinical judgment and } \\
\text { experience in simulation: An experimental } \\
\text { study. }\end{array}$ & $\begin{array}{l}\text { Nurse } \\
\text { Education } \\
\text { Today } \\
\text { International } \\
\text { Journal for } \\
\text { Health Care } \\
\text { Education }\end{array}$ & 2017 & Inglês \\
\hline 13 & Gaylle, D. & $\begin{array}{l}\text { In-simulation Debriefing Increases } \\
\text { Therapeutic Communication Skills }\end{array}$ & Nurse Educator & 2019 & Inglês \\
\hline 14 & I. smailog lu, E. G., Zaybak, A. & $\begin{array}{l}\text { Comparison of the Effectiveness of a Virtual } \\
\text { Simulator With a Plastic Arm Model in } \\
\text { Teaching Intravenous Catheter Insertion } \\
\text { Skills }\end{array}$ & $\begin{array}{l}\text { Computadores, } \\
\text { Informática, } \\
\text { Enfermagem }\end{array}$ & 2018 & Inglês \\
\hline 15 & $\begin{array}{l}\text { Donnelly, M. B., Horsley, T. L., } \\
\text { Adms, W, H., Gallagher, P., \& } \\
\text { Zibricky, C, D. }\end{array}$ & $\begin{array}{l}\text { Effect of simulation on Undergraduate } \\
\text { Nursing Students' Knowledge of Nursing } \\
\text { Ethics Principles. }\end{array}$ & Sage Journals & 2017 & Inglês \\
\hline 16 & $\begin{array}{l}\text { Ferri, P., Rovesti, S., Magnani, } \\
\text { D., Barbieri, A., Bargellini, A., } \\
\text { Mongelli, F., Bonetti, L., Vestri, } \\
\text { A., Alunni, D, F., \& Di Lorenzo, } \\
\text { R. }\end{array}$ & $\begin{array}{l}\text { A eficácia da simulação interprofissional na } \\
\text { melhoria da atitude colaborativa entre } \\
\text { estudantes de enfermagem e residentes de } \\
\text { medicina. Um protocolo de estudo para um } \\
\text { ensaio clínico randomizado }\end{array}$ & Acta Biomedica & 2018 & Português \\
\hline 17 & Gönenç, I, M., \& Sezer, Y, N. & $\begin{array}{l}\text { Evaluation of the effectiveness of four } \\
\text { different training techniques in the } \\
\text { development of non-stress testing application } \\
\text { skills: A randomised controlled trial. }\end{array}$ & Science Direct & 2019 & Inglês \\
\hline
\end{tabular}

Fonte: Autores (2021).

\section{Resultados e Discussão}

Os 17 artigos analisados estavam distribuídos em 14 periódicos, tendo um predomínio das Nursing Education Perspectives (2), Nurse Education Today (2) e REME - REVISTA MINEIRA DE ENFERMAGEM (2), respectivamente. Em relação ao ano, o maior número de publicações ocorreu no ano de 2020 (29,4\%) (Figura 2). Quanto ao tipo de língua, o inglês lidera com $12(70,6 \%)$ enquanto em português foram $5(29,4 \%)$. 
Figura 2: Quantidade de estudos publicados de acordo com o ano, sobre o uso da simulação na formação de enfermeiros.

\section{PUBLICAÇÕES POR ANO}

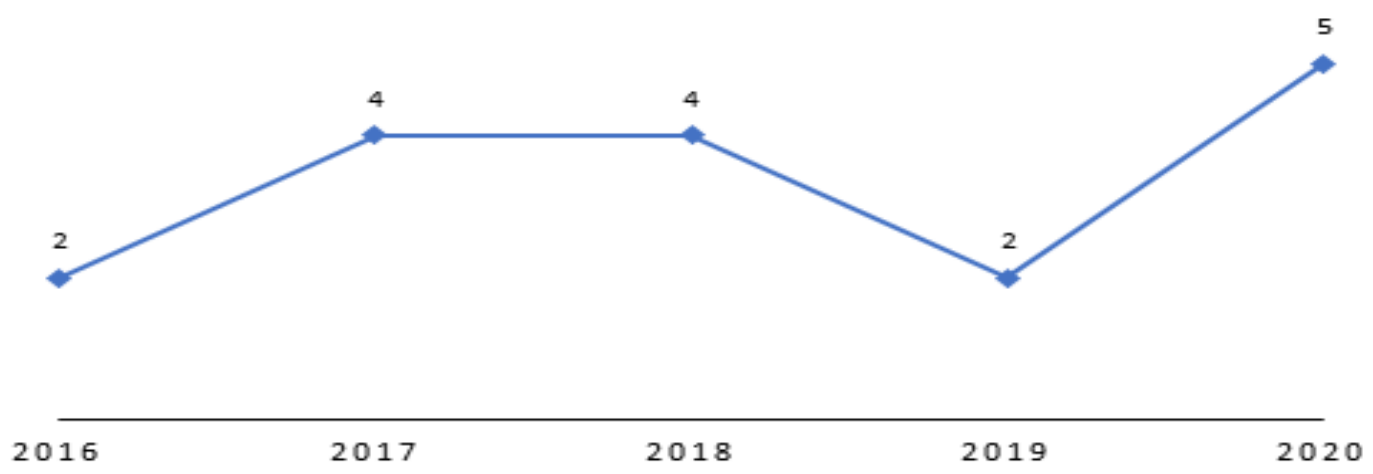

Fonte: Autores (2021).

Para melhor explanação dos resultados encontrados, os mesmos foram separados em categorias, que serão descritas a seguir.

\subsection{Identificação das principais técnicas utilizadas}

No ensino tradicional, o conhecimento é transmitido ao aluno de forma mais verbalista e teórica, sendo mais comuns em salas de aula ou treinamento em grupo. (Salgado et al., 2018). Já na simulação realística, além da aula teórica, o aluno tem aulas em um ambiente controlado com manequins que simulam o corpo humano, auxiliando o estudante na aprendizagem prática, antes de realizar determinado procedimento em pacientes. (Alamrani et al., 2018).

Dentro da simulação realística, podem ser utilizados manequins de alta fidelidade, baixa fidelidade e até o uso do debriefing. O debriefing consiste em uma reunião após a simulação, onde os estudantes podem discutir seus erros e ver onde estão falhando, desta forma aprendem para não repeti-los novamente. (Costa et al., 2020).

A partir dos estudos analisados, foram encontrados como técnicas (Quadro 2) a utilização de manequins (simulador paciente) em nove estudos $(52,9 \%)$, cinco $(29,4 \%)$ se referem ao uso de pessoas no papel de paciente (paciente simulado), dois $(11,8 \%)$ utilizam mais de uma forma de simulação (métodos mistos) e um $(5,9 \%)$ usou objetos virtuais de aprendizagem (softwares educativos).

Quadro 2: Tipos de técnicas utilizadas no ensino de enfermagem 2016-2020.

\begin{tabular}{|c|c|}
\hline Técnica Utilizada & $\mathbf{N}^{\circ}$ Artigo \\
\hline Simulador de paciente (manequim) alta fidelidade & $5,6,8,9,10,11,12,16$ \\
\hline Simulador de paciente (manequim) baixa fidelidade & 7 \\
\hline Simulador virtual & 3,17 \\
\hline Métodos mistos & $1,2,4,13,15$ \\
\hline Paciente Simulado & \\
\hline
\end{tabular}

Fonte: Autores (2021).

O estudo (3) realizado com estudantes de Enfermagem, em 4 áreas de especialidade clínica: obstetrícia, pediatria, saúde mental e cuidados intensivos. Teve como foco avaliar a eficácia do uso de simulações de alta fidelidade para substituir $50 \%$ das experiências clínicas tradicionais e para isso utilizou manequins e role-playing (interpretação de papéis) oferecendo oportunidades para que os discentes pudessem aplicar conhecimentos didáticos e desenvolver competências e habilidades de 
enfermagem. Esse trabalho indica que a combinação de simulações com experiências clínicas convencionais resultou significativamente mais pontuações no exame de saída da pré-graduação do que apenas experiências clínicas tradicionais (Tabela 2).

O uso do Simulador Virtual (14) foi utilizado em um simulador intravenoso com um modelo de braço de plástico no ensino de habilidades de inserção de cateter intravenoso para estudantes de enfermagem. A partir da análise dos estudantes frente a simulação virtual, o aluno é levado a tomar decisões clínicas escolhendo alternativas de condutas.

A simulação clínica tem um grande diferencial, quando há o momento reflexivo que acontece após a execução da cena e da prática realizada que é chamado de debriefing $(1,2,6,7,9,13)$. Esse espaço de descrição, discussão e síntese é um ponto essencial para assimilação e confiança tanto em competências técnicas quanto interpessoais para um resultado clínico eficaz.

\subsection{Descrição das vantagens do uso de Simulação Realística ao comparar-se a metodologia tradicional}

Ao analisar o uso da simulação realística empregado nos estudos, verificou-se que os alunos dessa metodologia, aumentaram o uso do pensamento crítico, trabalho em equipe, resolução de problemas e torna o estudante ativo no seu processo de conhecimento, gerando mais auto confiança e estimulando a criatividade do aluno. Na simulação, o ambiente é controlado e protegido contra possíveis danos, fazendo com que o participante consiga aprender sem medo antes de praticar no paciente propriamente dito.

Já no método tradicional, como consistem em basicamente aulas teóricas, podem gerar algum grau de ansiedade no aluno, visto que ele não terá uma prática em um ambiente seguro, que simule a realidade, então não terá uma experiência prévia, tendo como sua primeira prática, o paciente. Sendo assim, é possível afirmar que a grande maioria dos artigos apresentaram que os alunos que possuíam a experiência de simulação realística, tiveram melhores resultados do que os alunos do método tradicional, no entanto em alguns artigos foi abordado que não houveram diferenças significativas na comparação dos escores de grupo controle e experimental. (Costa et al., 2020; Ismailoglu et al., 2018; Donelly et al., 2017).

A partir dos dados analisados, foi possível identificar que os estudantes sempre se mostraram bastante satisfeitos com o uso da simulação realística como forma de aprendizagem, uma vez que o estudante participa de modo mais ativo e dinâmico do processo facilitando assim a sua aprendizagem além de conseguir correlacionar de modo mais claro a teoria com a prática e fazer uma melhor associação. (Costa et al., 2020).

Um dos fatores mais citados entre os estudantes, foi o aumento da autoconfiança, pois essa prática os estimula a refletir e a partir disso pensarem em estratégias possíveis para solucionar o caso, aumentando assim suas habilidades e pensamento crítico.

Contudo, deve-se levar em consideração o fato de estudantes que não conhecem a prática da simulação realística. Devendo então, esse assunto ser mais difundido para que aumente a adesão a essa prática.

Destacam-se algumas dificuldades para implementar a simulação realística como uma estratégia de ensino em um maior número de instituições: muitos professores não conhecerem ainda o verdadeiro benefício dessa prática, não terem interesse pela temática e nem capacitação para aplicá-la. Ademais, o espaço para a realização dessas práticas realísticas é um fator de importância que necessita ser mais destacado em pesquisas a fim de estimular o desenvolvimento de contextos e cenários de simulação realística no ambiente de ensino de enfermagem. (Costa et al., 2020)

\subsection{Identificadores de eficácia}

De acordo com os resultados obtidos com os artigos, a eficácia do ensino com a simulação realística trouxe mais benefícios para os estudantes, tornando uma melhor estratégia de aprendizagem. (Major et al., 2020).

Dessa forma, o aluno consegue desenvolver suas habilidades, seu senso crítico, não permite que sinta insegurança, 
facilita o trabalho em equipe e consequentemente isso o torna um melhor profissional, que consegue lidar com diversas situações através das suas vivências em seu ambiente de trabalho. Ademais, faz com que a aproximação da realidade aumente a capacidade de intervenção imediata após a identificação de algum problema e soluções em prol de melhores resultados. (Major et al., 2020).

\section{Conclusão}

Neste estudo foram identificados que os principais elementos utilizados como estratégia pela simulação realística foram: simulador de paciente (manequim) de alta fidelidade e paciente simulado. Pôde-se identificar que as principais vantagens do uso de simulação realística são: desenvolvimento de habilidades psicomotoras, resolução de problemas através de raciocínio clínico e crítico, priorização das atividades, melhora na comunicação entre integrantes da equipe, melhora no desempenho e rendimento dos estudantes, acreditando que os conhecimentos obtidos são mais difíceis de serem esquecidos, tornando os alunos melhor capacitados para a futura atuação profissional, além disso, os autores destacam uma maior confiança e satisfação por parte alunos que realizaram o ensino por simulação em função de serem participantes ativos desse processo e realizarem práticas em ambiente controlado e protegido que fizessem uma aproximação do que seria o cenário real com possibilidade de correção de erros. Tornando essa uma metodologia bastante eficaz no ensino de graduação para as áreas da saúde, destacando entre elas a enfermagem.

De acordo com a pergunta PICO utilizada para a elaboração do artigo "Quais as contribuições no processo de ensino aprendizagem causadas pela prática de simulação realística em comparação ao ensino tradicional em alunos da graduação de enfermagem?" e após o que foi exposto, notamos que a simulação realística possui diversas vantagens e contribuições se comparada ao método tradicional de ensino, tao como permitir o aluno desenvolver o pensamento crítico através de experiências controladas e seguras.

A partir da análise realizada, foi evidenciado que existem muitos trabalhos os quais abordam a simulação realística, no entanto, quando realizada a busca por comparação do método tradicional de ensino com o método de simulação realística para estudantes de graduação de enfermagem, o resultado ainda se encontra em menor número, sendo assim, sugere-se que esse tema seja foco de trabalhos futuros com o objetivo de ampliar a discussão sobre a percepção dos alunos que realizam a simulação realística antes de procedimentos e o que os mesmos caracterizam como positivo, possibilitando um entendimento maior acerca desse ponto de vista.

\section{Referências}

Akobeng, A. K. (2005). Principles of evidence based medicine. Archives of Disease in Childhood, 90(8), 837-840. https://doi.org/10.1136/adc.2005.071761

Alamrani, M. H., Alammar, K. A., Alqahtani, S. S., \& Salem, O. A. (2018). Comparing the Effects of Simulation-Based and Traditional Teaching Methods on the Critical Thinking Abilities and Self-Confidence of Nursing Students. The journal of nursing research. JNR, $26(3)$, $152-157$. https://doi.org/10.1097/jnr.0000000000000231

Alves, N. P., Gomes, T. G., Lopes, M. M. C. O., Gubert, F. A., Lima, M. A., Beserra, E. P., Martins, M. C., \& Cavalcante, V. M. V. (2019). Simulação realística e seus atributos para a formação do Enfermeiro. Rev enferm UFPE on line, 13 (5), 1420-1428. https://doi.org/10.5205/1981-8963v13i05a239014p1420-1428-2019. https://periodicos.ufpe.br/revistas/revistaenfermagem/article/view/239014/32337.

Baptista, R. C. N., Paiva, L. A. R., Gonçalves, R. F. L., Oliveira, L. M. N., Pereira, M. F. C. R., \& Martins, J. C. A. (2016). Satisfaction and gains perceived by nursing students with medium and high-fidelity simulation: A randomized controlled trial. Nurse Education Today, 46, 127-132. 10.1016/j.nedt.2016.08.027. https://pubmed.ncbi.nlm.nih.gov/27639211/

Barreto, D. G., Silva, K. G. N., Moreira, S. S. C. R., Silva, T. S., \& Magro, M. C. S. (2014). Simulação realística como estratégia de ensino para o curso de graduação em enfermagem: revisão integrativa. Revista Baiana de Enfermagem, 28 (2), 208-214. https:/www.google.com/url?sa=t\&rct=j\& $\mathrm{q}=\&$ esrc=s\&source=web\&cd=\&ved=2ahUKEwjw1cGjmrbyAhWypJUCHWoYB7gQFnoECAMQAQ\&url=https $\% 3 \mathrm{~A} \% 2 \mathrm{~F} \% 2 \mathrm{Fperiodicos}$. ufba.br\%2Findex.p hp\%2Fenfermagem\%2Farticle\%2Fdownload\%2F8476\%2F8874\&usg=AOvVaw1N93jIA6_BVhUOR5fW2c4g

Boostel, R., Felix, J., Bortolato-Major, C., Pedrolo, E., \& Vayego, S. (2018). Stress of nursing students in clinical simulation: a randomized clinical trial. Rev. Bras. Enferm, 71, 967-974. https://doi.org/10.1590/0034-7167-2017-0187. 
Bortolato-Majo, C., Mantovani, M. d. F., Felix, J. V. C., Boostel, R., \& Mattei, S. H. (2020). Autoconfiança e satisfação dos estudantes de enfermagem em simulação de emergência. REME - Rev Min Enferm, 24(1336). https://doi.org/10.5935/1415.2762.20200073

Brighente, M. F., \& Mesquida, P. (2016). Paulo Freire: da denúncia da educação bancária ao anúncio de uma pedagogia libertadora. Pro-Posições, 27 (1), 155177. 10.1590/0103-7307201607909. http://www.scielo.br/scielo.php?script=sci_arttext\&pid=S0103-73072016000100155\&lng=en\&nrm=iso.

Brooke, G., \& Jackie, C. (2017). Using Simulation to Enhance Education in an Obstetrical Nursing Course. The Journal of Nursing Research, 31(1), 40-41. 0.1097/01.NEP.0000000000000099.https://journals.lww.com/neponline/Abstract/2017/01000/Using_Simulation_to_Enhance_Education_in_an.11.aspx.

Cooper, J. B., \& Taqueti, V. R. (2008). A brief history of the development of mannequin simulators for clinical education and training. Postgraduate Medical Journal. 84, 563-570. https://doi.org/10.1136/qshc.2004.009886. https://pmj.bmj.com/content/84/997/563.short

Costa, R. R. O., Medeiros, S. M., Martins, J. C. A., Coutinho, V. R. D., \& Araújo, M. S. (2020). Eficácia da simulação no ensino de imunização em enfermagem: ensaio clínico randomizado. Rev. Latino-Am. Enfermagem, 28, 1-10. 10.1590/1518-8345.3147.3305. https://www.scielo.br/j/rlae/a/FQKbhgrZC3zX4Fp9SmNX8Rz/?lang=pt

Costa, R. R. O., Medeiros, S. M., Coutinho V. R. D., Mazzo, A., \& Araújo, M. S. (2020). Satisfação e autoconfiança na aprendizagem de estudantes de enfermagem: Ensaio clínico randomizado. Escola Anna Nery, 24 (1), 1-9. 10.1590/2177-9465-EAN-2019-0094. https://www.scielo.br/j/ean/a/mpbLpLMv6TNZ6fWRnHD9Jtx/?lang=pt

Curl, E. D., Smith, S., Chisholm, L. A., McGee, L. A., \& Das, K. (2016). Effectiveness of Integrated Simulation and Clinical Experiences Compared to Traditional Clinical Experiences for Nursing Students. Nursing Education Perspectives, $37 \quad$ (2), $72-77 . \quad 10.5480 / 15-1647$. https://pubmed.ncbi.nlm.nih.gov/27209864/

Donnelly, M. B., Horsley, T. L., Adams, W. H., Gallagher, P., \& Zibricky, C. D. (2017). Effect of Simulation on Undergraduate Nursing Students' Knowledge of Nursing Ethics Principles. The Canadian journal of nursing research = Revue canadienne de recherche en sciences infirmieres, 49(4), 153-159. https://doi.org/10.1177/0844562117731975. https://pubmed.ncbi.nlm.nih.gov/28948836/

Dourado, A. S. S., \& Giannella, T. R. (2014). Ensino baseado em simulação na formação continuada de médicos: análise das percepções de alunos e professores de um Hospital do Rio de Janeiro. Rev. bras. educ. med, 38(4), 460-469. https://doi.org/10.1590/S0100-55022014000400007

Ferreira, R. P., Guedes, H. M., Oliveira, D. W. D., \& Miranda, J. L. d. (2018). Simulação realística como método de ensino no aprendizado de estudantes da área da saúde. Revista de Enfermagem do Centro-Oeste Mineiro, 8, Artigo e2508. https://doi.org/10.19175/recom.v7i0.2508

Ferri, P. Rovesti, S. Magnani, D. Barbieri, A. Bargellini, A. Mongell, F. Bonetti, L.Vesttri, A. Allune, F. D. \& Di Lorenzo, R. (2018) The efficacy of interprofessional simulation in improving collaborative attitude between nursing students and residents in medicine. A study protocol for a randomised controlled trial. Acta Biomed. 20 (7-S):32-40. https://www.ncbi.nlm.nih.gov/pmc/articles/PMC6502140/

Gaba D. M. (2004). The future vision of simulation in health care. Quality and safety in health care.13 (1), 2-3. https://doi.org/10.1136/qhc.13.suppl_1.i2. https://www.ncbi.nlm.nih.gov/pmc/articles/PMC1765792/pdf/v013p000i2.pdf

Gadelha, M. M. T., Carmo, A. P., Andrade, M. E., Silva, J. M. A., Bezerra, I. C. B., \& Fernandes, M. C. (2020). Utilização das tecnologias educativas: distância oceânica entre o processo formativo real e o ideal na enfermagem. R. pesq.: cuid. fundam. Online, 12, 909-914. 10.9789/2175-5361.rpcfo.v12.7950. https://pesquisa.bvsalud.org/portal/resource/pt/biblio-1119067

Gaylle, D. (2019). In-simulation Debriefing Increases Therapeutic Communication Skills. Nurse Educ. 44 (6), 295-299. .https://journals.lww.com/nurseeducatoronline/Abstract/2019/11000/In_simulation_Debriefing_Increases_Therapeutic.6.aspx.

Gönenç, İ. M., \& Sezer, N. Y. (2019) four different training techniques in the development of non-stress testing application skills: A randomised controlled trial. Nurse Educ. (76), 118-124. https://www.sciencedirect.com/science/article/abs/pii/S0260691719301820

Institute of Medicine (US) Committee on Quality of Health Care in America. Kohn, L. T., Corrigan, J. M., \& Donaldson, M. S. (2000). To Err is Human: Building a Safer Health System. National Academies Press (US). https://pubmed.ncbi.nlm.nih.gov/25077248/

Ismailoglu, E G, \& Zaybak A. (2018). Comparison of the Effectiveness of a Virtual Simulator With a Plastic Arm Model in Teaching Intravenous Catheter Insertion Skills. CIN: Computers, Informatics, Nursing. $36 \quad$ (2), 98-105. https://journals.lww.com/cinjournal/Abstract/201 8/02000/Comparison_of_the_Effectiveness_of_a_Virtual.7.aspx

Jones, F., Passos-Neto, C. E., \& Braghiroli, O. F. M. (2015). Simulation in Medical Education: Brief history and methodology. Principles and Practice of Clinical Research, 1(2), 46-54. http://dx.doi.org/10.21801/ppcrj.2015.12.8. https://journal.ppcr.org/index.php/ppcrjournal/article/view/12

Kuo, S., Wu, J., Chen, H., Chen, C., \& Hu, S. H. (2020). Comparação dos efeitos do treinamento de simulação e cenários baseados em problemas na melhoria de estudantes de enfermagem graduados para falar sobre erros de medicação: um estudo quase experimental. Nurse Education Today,

Link, E. A. (2014). The National Center For Simulation [Site]. Recuperado de https:/www.simulationinformation.com/hall-of-fame/members/edwin-albertlink/

Martins, J. C. A., Mazzo, A., Baptista, R. C. N., Coutinho, V. R. D., Godoy, S., Mendes, I. A. C., \& Trevizan, M. A. (2012). A experiência clínica simulada no ensino de enfermagem: retrospectiva histórica. Acta paul. Enferm, 25 (4), 619-625. https://doi.org/10.1590/S0103-21002012000400022. http://www.scielo.br/scielo.php?script=sci arttext\&pid=S0103-21002012000400022\&lng=en\&nrm=iso

Martins, J. C. A. (2017). Aprendizagem e desenvolvimento em contexto de prática simulada. Revista de Enfermagem Referência, 12, 155-162. https://doi.org/10.12707/RIV16074. http://www.scielo.mec.pt/scielo.php?script=sci_arttext\&pid=S0874-02832017000100016\&lng=pt 
Research, Society and Development, v. 10, n. 14, e303101421956, 2021

(CC BY 4.0) | ISSN 2525-3409 | DOI: http://dx.doi.org/10.33448/rsd-v10i14.21956

Mendes, K. D. S., Silveira, R. C. C. P., \& Galvão, C. M. (2008). Revisão integrativa: método de pesquisa para a incorporação de evidências na saúde e na

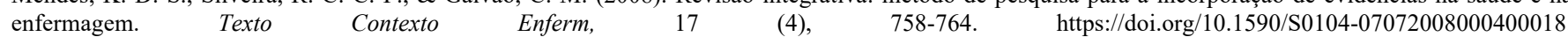
https://www.scielo.br/j/tce/a/XzFkq6tjWs4wHNqNjKJLkXQ/?lang=pt\&format=html

Miranda, F. B. G., Mazzo, A., \& Junior, G. A. P. (2018). Uso da simulação de alta fidelidade no preparo de enfermeiros para o atendimento de urgências e emergências: revisão da literatura. Sci Med, 28(1), Artigo 28675. https://doi.org/10.15448/1980-6108.2018.1.28675

Oliveira, S. N. d., Prado, M. L. d., \& Kempfer, S. S. (2014). Utilização da simulação no ensino da enfermagem: revisão integrativa. REME rev min enferm, 18(2), 487-95. https://doi.org/10.5935/1415-2762.20140036

Rosen, K. R. The history of medical simulation. (2008). Journal of Critical Care. (2a ed.). 23, 157-166. https://oi.org/10.1016/j.jcrc.2007.12.004. https://www.sciencedirect.com/science/article/abs/pii/S0883944107002018

Salgado, P. O., Souza, C. C., Júnior, P. P. P., Balbino, P. C., Ribeiro, L., Paiva, L. C., \& Brombine, N. L. M. (2018). O uso da simulação no ensino da técnica de aspiração de vias aéreas: Ensaio clínico randomizado controlado. Rev Min Enferm. 22, 1-9. 10.5935/1415-2762.20180020. https://cdn.publisher.gn1.link/reme.org.br/pdf/e1090.pdf.

Silva, A. N., Senna, M. A. A., Teixeira, M. C. B., Lucietto D. A., \& Andrade I. M. (2020). O uso de metodologia ativa no campo das Ciências Sociais em Saúde: relato de experiência de produção audiovisual por estudantes. Interface (Botucatu), 24, 1-14. https://doi.org/10.1590/Interface.190231. https://www.scielo.br/j/icse/a/9F3KQv5NHSwtPHN8qFhD4wr/?lang=pt

Souza, A. S. d. (2011). O projeto político pedagógico do Curso de Enfermagem da Universidade Federal de Pelotas. Rev. enferm. saúde, 1(1), 164-176. https://DOI.ORG/10.15210/JONAH.V1I1.3420

Veiga, G. A., Araújo, M. C., Cauduro, F. L. F., \& Andrade J. (2020). Metodologia ativa no estágio supervisionado de Enfermagem: inovação na atenção primária à saúde. Rev baiana enferm, 34, 1-9. 10.18471/rbe.v34.34857. https://pesquisa.bvsalud.org/portal/resource/pt/biblio-1098744

Yamane, M. T., Machado, V. K., Osternack, K. T., \& Mello, R. G. (2019). Simulação realística como ferramenta de ensino na saúde: uma revisão integrativa. Rev. Espaço para a Saúde, 20 (1), 87-107. 10.22421/15177130-2019v20n1p87. https://pesquisa.bvsalud.org/portal/resource/pt/biblio-1008011 